\title{
Evaluation of organic amendment on the effect of cadmium bioavailability in contaminated soils using the DGT technique and traditional methods
}

\author{
Yu Yao ${ }^{1} \cdot$ Qin Sun ${ }^{1} \cdot$ Chao Wang ${ }^{1} \cdot$ Pei-fang Wang ${ }^{1} \cdot$ Shi-ming Ding ${ }^{2}$
}

Published online: 21 March 2017

(C) Springer-Verlag Berlin Heidelberg 2017

\begin{abstract}
Organic amendments have been widely proposed as a remediation technology for metal-contaminated soils, but there exist controversial results on their effectiveness. In this study, the effect of pig manure addition on cadmium $(\mathrm{Cd})$ bioavailability in Cd-contaminated soils was systematically evaluated by one dynamic, in situ technique of diffusive gradients in thin films (DGT) and four traditional methods based on the equilibrium theory (soil solution concentration and the three commonly used extractants, i.e., acetic acid (HAc), ethylenediamine tetraacetic acid (EDTA), and calcium chloride $(\mathrm{CaCl} 2)$. Wheat and maize were selected for measurement of plant $\mathrm{Cd}$ uptake. The results showed that pig manure addition could promote the growth of two plants, accompanied by increasing biomasses of shoots and roots with increasing doses of pig manure addition. Correspondingly, increasing additions of pig manure reduced plant $\mathrm{Cd}$ uptake and accumulation, as indicated by the decreases of $\mathrm{Cd}$ concentrations in shoots and roots. The bioavailable concentrations of $\mathrm{Cd}$ in $\mathrm{Cd}-$ contaminated soils reflected by the DGT technique obviously decreased with increasing doses of pig manure addition,
\end{abstract}

Qin Sun

sunqinnj@hhu.edu.cn

Key Laboratory of Integrated Regulation and Resource Development on Shallow Lakes, Ministry of Education, College of Environment, Hohai University, Nanjing 210098, China

2 State Key Laboratory of Lake Science and Environment, Nanjing Institute of Geography and Limnology, Chinese Academy of Sciences, Nanjing 210008, China following the same changing trend as plant Cd uptake. Changes in soil solution $\mathrm{Cd}$ concentration and extractable $\mathrm{Cd}$ by $\mathrm{HAc}$, EDTA, and $\mathrm{CaCl} 2$ in soils were similar to DGT measurement. Meanwhile, the capability of Cd resupply from solid phase to soil solution decreased with increasing additions of pig manure, as reflected by the decreases in the ratio (R) value of CDGT to Csol. Positive correlations were observed between various bioavailable indicators of $\mathrm{Cd}$ in soils and $\mathrm{Cd}$ concentrations in the tissues of the two plants. These findings provide stronger evidence that pig manure amendment is effective in reducing Cd mobility and bioavailability in soils and it is an ideal organic material for remediation of Cdcontaminated soils.

This article (DOI 10.1007/s11356-015-5218-8) was originally intended as an invited contribution to the special issue Bioavailability - the underlying basis for Risk Based Land Management that was published in Environmental Science and Pollution Research Volume 22 Issue 12. 